\title{
A THEOREM ON POLYNOMIAL-STAR APPROXIMATION
}

\author{
A. M. DAVIE AND T. W. GAMELIN \\ (Communicated by Paul S. Muhly)
}

\begin{abstract}
We prove that the unit ball of a Banach space is polynomial-star dense in the unit ball of its bidual. This strengthens Goldstine's theorem on weak-star density.
\end{abstract}

\section{SUMmary}

Let $\mathscr{X}$ be a Banach space, with bidual $\mathscr{X}^{* *}$. Goldstine's theorem [5] asserts that the open unit ball $B$ of $\mathscr{X}$ is weak-star dense in the unit ball $B^{* *}$ of $\mathscr{X}^{* *}$. In other words, for any $z \in \mathscr{X}^{* *}$ with $\|z\| \leq 1$, there is a net $x_{\alpha} \in$ $B$ with $L\left(x_{\alpha}\right) \rightarrow z(L)$ for all $L \in \mathscr{X}^{*}$. Our aim is to show that the net $\left\{x_{\alpha}\right\}$ can be chosen so that $P\left(x_{\alpha}\right)$ converges to a certain value $\widehat{P}(z)$ for any analytic polynomial $P$ on $\mathscr{X}$. It will follow then that every $f \in H^{\infty}(B)$ extends in a canonical fashion to a function $\hat{f} \in H^{\infty}\left(B^{* *}\right)$, to give an isometric algebra embedding of $H^{\infty}(B)$ as a closed subalgebra of $H^{\infty}\left(B^{* *}\right)$. This settles a problem left open by Aron and Berner [1], who had shown that the canonically associated extension $\hat{f}$ of $f \in H^{\infty}(B)$ is analytic in the ball in $\mathscr{X}^{* *}$ centered at 0 of radius $1 / e$.

\section{STATEMENT OF THE MAIN THEOREM}

Recall that an $m$-homogeneous analytic polynomial $P$ on $\mathscr{Z}$ is the restriction to the diagonal of a continuous (complex-valued) $m$-linear form $F$ on $\mathscr{Z}$ :

$$
P(x)=F(x, \ldots, x), \quad x \in \mathscr{X} .
$$

Each such $P$ is the restriction of a unique symmetric $m$-linear form on $\mathscr{Z}$, and the symmetric extension $F$ can be recovered from $P$ by the usual polarization formula $[4,6]$. We denote

$$
\|P\|=\sup \{|P(x)|: x \in B\}
$$

and

$$
\|F\|=\sup \left\{\left|F\left(x_{1}, \ldots, x_{m}\right)\right|: x_{1}, \ldots, x_{m} \in B\right\} .
$$

Received by the editors June 6, 1988 .

1980 Mathematics Subject Classification (1985 Revision). Primary 46G20; Secondary 30H05.

The second author was partially supported by NSF Grant \#DMS85-03780. 
The polarization formula yields immediately the estimate

$$
\|F\| \leq \frac{m^{m}}{m !}\|P\|,
$$

and this estimate is sharp [6].

Each continuous $m$-linear form $F$ on $\mathscr{Z}$ can be extended to an $m$-linear form on the bidual $\mathscr{Z}^{* *}$, generally in many ways. One such extension, which we denote by $\widehat{F}$, is obtained by specifying that for each fixed $j, 1 \leq j \leq m$, and for each fixed $x_{1}, \ldots, x_{j-1} \in \mathscr{X}$ and $z_{j+1}, \ldots, z_{m} \in \mathscr{X}^{* * *}$, the linear functional

$$
z \rightarrow \widehat{F}\left(x_{1}, \ldots, x_{j-1}, z, z_{j+1}, \ldots, z_{n}\right), \quad z \in \mathscr{X}^{* *},
$$

is weak-star continuous. In other words, one extends by weak-star continuity, beginning with the last variable and working backwards to the first. If one takes the variables in a different order for purposes of extending, one generally arrives at a different extension. However, the restriction

$$
\widehat{P}(z)=\widehat{F}(z, \ldots, z), \quad z \in \mathscr{X}^{* *},
$$

of $\widehat{F}$ to the diagonal is independent of the order in which the variables are treated, and evidently

$$
\|\widehat{P}\| \leq\|\widehat{F}\|=\|F\| \leq \frac{m^{m}}{m !}\|P\| .
$$

The correspondence $P \rightarrow \widehat{P}$ is multiplicative. It extends to an algebra isomorphism of the analytic polynomials on $\mathscr{X}$ and an algebra of analytic polynomials on $X^{* *}$. For details, see [1].

Our main theorem is the following.

Theorem 1. Let $S$ be a bounded subset of $\mathscr{X}$, and suppose $z \in X^{* *}$ is weakstar adherent to $S$. Then there is a net $\left\{x_{\alpha}\right\}$ in $\mathscr{X}$ such that each $x_{\alpha}$ is an arithmetic mean of distinct elements of $S$, and $P\left(x_{\alpha}\right) \rightarrow \widehat{P}(z)$ for all analytic polynomials $P$ on $\mathscr{X}$.

For purposes of stating more succinctly the result, we define the polynomialstar topology of $\mathscr{X}^{* *}$ to be the smallest topology for which a net $\left\{z_{\alpha}\right\}$ converges to $z$ if and only if $\widehat{P}\left(z_{\alpha}\right)$ converges to $\widehat{P}(z)$ for all analytic polynomials $P$ of $\mathscr{X}$. Evidently norm convergence implies polynomial-star convergence, and since continuous linear functionals are in particular analytic polynomials, polynomial-star convergence implies weak-star convergence. As an immediate consequence of Theorem 1 we obtain the following.

Theorem 2. Let $S$ be a bounded convex subset of $\mathscr{X}$. Then the weak-star closure of $S$ in $\mathscr{X}^{* *}$ coincides with the polynomial-star closure of $S$ in $\mathscr{X}^{* *}$.

In particular, the unit ball $B$ of $\mathscr{Z}$ is polynomial-star dense in the unit ball $B^{* *}$ of $\mathscr{X}^{* *}$. This is our extension of Goldstine's theorem. 
Note that Theorem 2 is trivial if $\mathscr{Z}$ is reflexive, since then the weak-star (=weak) closure of $S$ coincides with the norm closure, even without the hypothesis of boundedness of $S$.

As another immediate application of Theorem 1, applied to the unit ball of $\mathscr{Z}$, we obtain the following sharpened form of the estimate (2.1).

Theorem 3. If $P$ is an analytic polynomial on $\mathscr{X}$, and $\widehat{P}$ is its canonical extension to $\mathscr{X}^{* *}$, then

$$
\|P\|_{B}=\|\widehat{P}\|_{B^{* *}} .
$$

Before giving the proof of Theorem 1, we make two observations.

It is essential that one be allowed to pass to arithmetic means in Theorem 1. To see this, let $S$ be an orthogonal basis $\left\{e_{j}\right\}_{j=1}^{\infty}$ for $l^{2}$. For $t=\sum t_{j} e_{j} \in l^{2}$, define $P(t)=\sum t_{j}^{2}$. Then $P$ is a 2 -homogeneous analytic function on $l^{2}$. Since $P\left(e_{j}\right)=1$ does not converge to $P(0)=0,0$ does not lie in the polynomial-star closure of $S$. However, since $e_{j} \rightarrow 0$ weakly, 0 lies in the weak (=weak-star) closure of $S$.

For some Banach spaces (such as $l^{p}, 1 \leq p<\infty$ ), it can be shown that a sequence converges in the polynomial-star topology if and only if it converges in norm. For this and related results, see [3]. Thus Theorem 1 is analogous to the theorem, valid in some Banach spaces, that every weakly convergent sequence has a subsequence whose arithmetic means converge in norm.

\section{Proof of Theorem 1}

The proof will depend on the following lemma.

Lemma. Let $z \in X^{* *}$, and let $S$ be a subset of $\mathscr{X}$ which contains $z$ in its weak-star closure. Let $\mathscr{F}$ be a finite family of continuous symmetric multilinear forms on $\mathscr{X}$. Let $\varepsilon>0$ and $N \geq 1$. Then there exist $x_{1}, \ldots, x_{N} \in S$ such that

$$
\left|F\left(x_{i_{1}}, \ldots, x_{i_{m}}\right)-\widehat{F}(z, \ldots, z)\right|<\varepsilon,
$$

whenever $F \in \mathscr{F}$ is an $m$-form and $i_{1}, \ldots, i_{m}$ are distinct indices between 1 and $N$.

Proof. Since each $F \in \mathscr{F}$ is symmetric, it suffices to obtain the estimate when $i_{1}<\cdots<i_{m}$. Let $\varepsilon^{\prime}>0$ be small. The $x_{j}$ 's are selected inductively by the following procedure, which depends on the weak-star continuity property of the $\widehat{F}$ 's described earlier.

First we choose $x_{1} \in S$ such that

$$
\left|\widehat{F}\left(x_{1}, z, \ldots, z\right)-\widehat{F}(z, z, \ldots, z)\right|<\varepsilon^{\prime}, \quad F \in \mathscr{F} .
$$

Then we chose $x_{2} \in S$ such that

$$
\left|\widehat{F}\left(x_{2}, z, \ldots, z\right)-F(z, \cdots, z)\right|<\varepsilon^{\prime}, \quad F \in \mathscr{F},
$$

and moreover,

$$
\left|\widehat{F}\left(x_{1}, x_{2}, z, \ldots, z\right)-\widehat{F}\left(x_{1}, z, z, \ldots, z\right)\right|<\varepsilon^{\prime}, \quad F \in \mathscr{F} .
$$


Proceeding in this fashion, we obtain $x_{j}$ 's so that

$$
\begin{aligned}
\mid \widehat{F}\left(x_{i_{1}}, \ldots, x_{i_{r-1}}, x_{i_{r}}, z, \ldots, z\right) & \\
& -\widehat{F}\left(x_{i_{1}}, \ldots, x_{i_{r-1}}, z, z, \ldots, z\right) \mid<\varepsilon^{\prime}, F \in \mathscr{F},
\end{aligned}
$$

whenever $i_{1}<\cdots<i_{r}$. Then

$$
\left|F\left(x_{i_{1}}, \ldots, x_{i_{m}}\right)-\widehat{F}(z, \ldots, z)\right|
$$

is estimated by a sum of $m$ terms

$\left|F\left(x_{i_{1}}, \ldots, x_{i_{m}}\right)-\widehat{F}\left(x_{i_{1}}, \ldots, x_{i_{m-1}}, z\right)\right|+\cdots+\left|\widehat{F}\left(x_{i_{1}}, z, \ldots, z\right)-\widehat{F}(z, \ldots, z)\right|$,

each smaller than $\varepsilon^{\prime}$. This is less than $\varepsilon$, for all $F \in \mathscr{F}$, providing $\varepsilon^{\prime}$ is sufficiently small.

Now to prove Theorem 1 , let $\varepsilon>0$, and let $\mathscr{F}$ be a finite family of continuous symmetric multilinear forms on $\mathscr{X}$. It suffices to find an arithmetic mean $x$ of elements of $S$ such that

$$
|\widehat{F}(z, \ldots, z)-F(x, \ldots, x)| \leq 2 \varepsilon
$$

for all $F \in \mathscr{F}$. For this, fix $N$ large, choose $x_{1}, \ldots, x_{N} \in S$ as in the lemma, and set

$$
x=\left(x_{1}+\cdots+x_{N}\right) / N .
$$

If $F \in \mathscr{F}$ is $m$-linear, we express

$$
\begin{aligned}
F(x, \ldots, x)-\widehat{F}(z, \ldots, z) & =\frac{1}{N^{m}} \sum_{i_{1}, \ldots, i_{m}=1}^{N}\left[F\left(x_{i_{1}}, \ldots, x_{i_{m}}\right)-\widehat{F}(z, \ldots, z)\right] \\
& =\Sigma_{1}+\Sigma_{2},
\end{aligned}
$$

where $\Sigma_{1}$ is the sum over $m$-tuples of distinct indices, and $\Sigma_{2}$ is the sum over the remaining indices. From the lemma we obtain

$$
\left|\Sigma_{1}\right|<\varepsilon \text {. }
$$

Since there are $N^{m}-N(N-1) \cdots(N-m+1)$ summands in $\Sigma_{2}$, each bounded by a constant $C$ depending only on $\mathscr{F}$ and $S$, we obtain

$$
\left|\Sigma_{2}\right| \leq\left[1-\left(1-\frac{1}{N}\right) \cdots\left(1-\frac{m-1}{N}\right)\right] C \text {. }
$$

For $N$ sufficiently large this is also less than $\varepsilon$, so that we obtain the required estimates above.

4. EMBEDDING $H^{\infty}(B)$ IN $H^{\infty}\left(B^{* *}\right)$

Consider a Taylor series

$$
\sum_{m=0}^{\infty} P_{m}(x)
$$


where $P_{m}$ is an $m$-homogeneous analytic polynomial on $\mathscr{X}, m \geq 0$. Recall that the radius of boundedness of the series is the largest $R, 0 \leq R \leq+\infty$, such that the series converges and represents a bounded analytic function on any ball $B_{r}=r B$ of radius $r<R$. In this case, the series (4.1) converges uniformly on each ball $B_{r}, r<R$. It is easy to check $[4,6]$ that the radius of boundedness is given by the expression

$$
R=1 / \lim \sup \left\|P_{m}\right\|^{1 / m} .
$$

We may also form the series

$$
\sum_{m=0}^{\infty} \widehat{P}_{m}(z)
$$

on $\mathscr{X}^{* *}$, for which the radius of boundedness is obtained by replacing $P_{m}$ by $\widehat{P}_{m}$ in (4.2). From Theorem 3 we then obtain immediately the following.

Theorem 4. The radius of boundedness of the Taylor series (4.1) in $\mathscr{Z}$ coincides with that of the series (4.3) in $\mathscr{Z}^{* *}$.

If now $f$ is given by the Taylor series (4.1), we define $\widehat{f}$ to be the sum of the Taylor series (4.3). Note that $\widehat{f}$ is bounded on each ball $B_{r}^{* *}=r B^{* *}$, when $r$ is strictly less than the radius of boundedness of (4.1).

Lemma. Suppose $f$ has Taylor series given by (4.1) with radius of boundedness $R>0$, and that $0<r<R$. Let $\left\{x_{\alpha}\right\}$ be a net in $B_{r}$ converging to $z \in \mathscr{X}^{* *}$ in the polynomial-star topology. Then $\left\{f\left(x_{\alpha}\right)\right\}$ converges to $\hat{f}(z)$.

Proof. Fix $\varepsilon>0$. Let $N \geq 1$, and let $Q$ be the $N$ th partial sum of the series (4.1). Then $\hat{Q}$ is the $N$ th partial sum of the series (4.3) for $\hat{f}$. Since the radius of boundedness of (4.3) is $R>r$, we can choose $N$ so large that

$$
\|\hat{f}-\hat{Q}\|_{B_{r}^{*}}<\varepsilon \text {. }
$$

Since $\widehat{Q}=Q$ on $\mathscr{X}$, we obtain

$$
\left|f\left(x_{\alpha}\right)-\hat{f}(z)\right| \leq\left|f\left(x_{\alpha}\right)-Q\left(x_{\alpha}\right)\right|+\left|Q\left(x_{\alpha}\right)-\hat{Q}(z)\right|+|\hat{Q}(z)-\hat{f}(z)| .
$$

Since $Q\left(x_{\alpha}\right) \rightarrow \widehat{Q}(z)$, we obtain

$$
\lim \sup \left|f\left(x_{\alpha}\right)-\hat{f}(z)\right| \leq 2 \varepsilon .
$$

This establishes the lemma.

Theorem 5. If $f \in H^{\infty}(B)$ has Taylor series (4.1), then the series (4.3) converges on $B^{* *}$ to a function $\hat{f} \in H^{\infty}(B)$, satisfying

$$
\|\hat{f}\|_{B^{*}}=\|f\|_{B} .
$$

The correspondence $f \rightarrow \hat{f}$ is an isometric isomorphism of $H^{\infty}(B)$ and a closed subalgebra of $H^{\infty}\left(B^{* *}\right)$.

Proof. The preceding lemma shows that

$$
|\hat{f}(z)| \leq\|f\|_{B}
$$


for all $z \in B^{* *}$, so that the correspondence $f \rightarrow \hat{f}$ is an isometry. Since the operator $P \rightarrow \widehat{P}$ is linear and multiplicative on analytic polynomials, and these are dense in $H^{\infty}(B)$ in the topology of uniform convergence on the $B_{r}$ 's, $0<r<1$, the correspondence is linear and multiplicative on $H^{\infty}(B)$.

It would be of interest to characterize the value $\hat{f}(z)$ of the canonical extension of $f \in H^{\infty}(B)$, without resorting to the Taylor series of $f$. From the point of view of the spectrum of $H^{\infty}(B)$, the problem is to determine what distinguishes the complex-valued homomorphisms which arise from points of $B^{* *}$ from the remaining ones. For more on the spectrum of $H^{\infty}(B)$, see [2].

\section{REFERENCES}

1. R. M. Aron and P. D. Berner, A Hahn-Banach extension theorem for analytic mappings, Bull. Soc. Math. France 106 (1978), 3-24.

2. R. M. Aron, B. J. Cole and T. W. Gamelin, Algebras of analytic functions on Banach spaces (in preparation).

3. T. K. Carne, B. J. Cole and T. W. Gamelin, $A$ uniform algebra of analytic functions on a Banach space, Trans. Am. Math. Soc., (to appear).

4. S. B. Chae, Holomorphy and calculus in normed spaces, Marcel Dekker, Inc., New York, 1985.

5. N. Dunford and J. Schwartz, Linear operators, Part I, Wiley, New York, 1964.

6. L. Nachbin, Topology on spaces of holomorphic mappings, Ergebnisse der Math., Vol. 47, Springer-Verlag, New York, 1969.

Department of Mathematics, University of Edinburgh, Edinburgh, E49 3JZ, Scotland

Department of Mathematics, UCla, Los Angeles, California 90024 